\title{
Microarray-Based Mutation Detection and Phenotypic Characterization of Patients with Leber Congenital Amaurosis
}

\author{
Suzanne Yzer, ${ }^{1,2,3}$ Bart P. Leroy, ${ }^{3,4,5}$ Elfride De Baere, ${ }^{5}$ Thomy J. de Ravel, ${ }^{6}$ \\ Marijke N. Zonneveld, ${ }^{2}$ Krysta Voesenek, ${ }^{2}$ Ulrich Kellner, ${ }^{7}$ Jose P. Martinez Ciriano, ${ }^{1}$ \\ Jan-Tjeerd H. N. de Faber, ${ }^{1}$ Klaus Robrschneider, ${ }^{8}$ Ronald Roepman, ${ }^{2}$ \\ Anneke I. den Hollander, ${ }^{2}$ Jobannes R. Cruysberg, ${ }^{9}$ Françoise Meire, ${ }^{4}$ Ingele Casteels, ${ }^{10}$ \\ Norka G. van Moll-Ramirez, ${ }^{11}$ Rando Allikmets, ${ }^{12}$ L. Ingeborgh van den Born, ${ }^{1}$ and \\ Frans P. M. Cremers ${ }^{2}$
}

Purpose. To test the efficiency of a microarray chip as a diagnostic tool in a cohort of northwestern European patients with Leber congenital amaurosis (LCA) and to perform a genotypephenotype analysis in patients in whom pathologic mutations were identified.

Methods. DNAs from 58 patients with LCA were analyzed using a microarray chip containing previously identified disease-associated sequence variants in six LCA genes. Mutations identified by chip analysis were confirmed by sequence analysis. On identification of one mutation, all protein coding exons of the relevant genes were sequenced. In addition, sequence analysis of the RDH12 gene was performed in 22 patients. Patients with mutations were phenotyped.

Results. Pathogenic mutations were identified in 19 of the 58 patients with LCA (32.8\%). Four novel sequence variants were identified. Mutations were most frequently found in CRB1 (15.5\%), followed by GUCY2D (10.3\%). The p.R768W muta-

From the ${ }^{1}$ The Rotterdam Eye Hospital, Rotterdam, The Netherlands; the Departments of ${ }^{2}$ Human Genetics and ${ }^{9}$ Ophthalmology, Radboud University Nijmegen Medical Centre, Nijmegen, The Netherlands; the ${ }^{4}$ Department of Ophthalmology and the ${ }^{5}$ Center for Medical Genetics, Ghent University Hospital, Ghent, Belgium; the ${ }^{6}$ Center for Human Genetics and the ${ }^{10}$ Department of Ophthalmology, Leuven University Hospitals, Leuven, Belgium; ${ }^{7}$ RetinaScience, Bonn, Germany; the ${ }^{8}$ Department of Ophthalmology, University of Heidelberg, Heidelberg, Germany; ${ }^{11}$ Sensis, Grave, The Netherlands; and the ${ }^{12}$ Department of Ophthalmology and Pathology, Columbia University, New York, New York.

${ }^{3}$ Contributed equally to the work and therefore should be considered equivalent authors.

Supported by the Flieringa/Stichting Wetenschappelijk Onderzoek Oogziekenhuis Rotterdam, the Bijzonder Onderzoeksfonds Universiteit Gent (Verkennend Europees Onderzoeksproject 011V1602), the Fonds voor Research in Oftalmologie/Fonds pour la Recherche en Ophthalmologie Belgium.

Submitted for publication July 1, 2005; revised September 6, 2005; accepted January 23, 2006.

Disclosure: S. Yzer, None; B.P. Leroy, None; E. De Baere, None; T.J. de Ravel, None; M.N. Zonneveld, None; K. Voesenek, None; U. Kellner, None; J.P. Martinez Ciriano, None; J.-T.H.N. de Faber, None; K. Rohrschneider, None; R. Roepman, None; A.I. den Hollander, None; J.R. Cruysberg, None; F. Meire, None; I. Casteels, None; N.G. van Moll-Ramirez, None; R. Allikmets, None; L.I. van den Born, None; F.P.M. Cremers, None

The publication costs of this article were defrayed in part by page charge payment. This article must therefore be marked "advertisement" in accordance with 18 U.S.C. $\$ 1734$ solely to indicate this fact.

Corresponding author: Frans P. M. Cremers, Department of Human Genetics, Radboud University Nijmegen Medical Centre, P.O. Box 9101, 6500 HB Nijmegen, The Netherlands; f.cremers@antrg.umcn.nl. tion was found in 8 of 10 GUCY2D alleles, suggesting that it is a founder mutation in the northwest of Europe. In early childhood, patients with AIPL1 or GUCY2D mutations show normal fundi. Those with AIPL1-associated LCA progress to an RP-like fundus before the age of 8 , whereas patients with GUCY2Dassociated LCA still have relatively normal fundi in their mid20s. Patients with CRB1 mutations present with distinct fundus abnormalities at birth and consistently show characteristics of RP12. Pathogenic GUCY2D mutations result in the most severe form of LCA.

Conclusions. Microarray-based mutation detection allowed the identification of $32 \%$ of LCA sequence variants and represents an efficient first-pass screening tool. Mutations in CRB1, and to a lesser extent, in GUCY2D, underlie most LCA cases in this cohort. The present study establishes a genotype-phenotype correlation for AIPL1, CRB1, and GUCY2D. (Invest Ophthalmol Vis Sci. 2006;47:1167-1176) DOI:10.1167/iovs.05-0848

$I^{2}$ 1869, Theodor Leber reported an "intrauterine" form of retinitis pigmentosa (RP). ${ }^{1}$ These children, mostly from consanguineous marriages, were congenitally blind without any retinal abnormalities. Later, many displayed a fundus picture typically found in other retinal dystrophies such as RP. This condition is now known as Leber congenital amaurosis (LCA), a clinically and genetically heterogeneous disorder characterized by severe bilateral congenital retinal blindness, with nystagmus and a nondetectable ERG before the age of 1 year. ${ }^{2}$

Thus far, mutations in seven genes (AIPL1, CRB1, CRX, GUCY2D, RDH12, RPE65, and RPGRIP1) have been shown to cause LCA, accounting for $35 \%$ to $47.5 \%$ of cases of LCA. ${ }^{3-12}$ Mutations in all these genes, except RPGRIP1, have also been found to cause other, clinically less severe retinal diseases, such as cone-rod dystrophy (CRD) or RP, with autosomal dominant inheritance shown in several cases. ${ }^{13,14}$

A specific subtype of RP with a distinct phenotype caused by mutations in the CRB1 gene is usually referred to as RP12. The clinical characteristics of this autosomal recessive disease include early central and peripheral visual loss, hyperopia, maculopathy, nummular rather than spicular pigmentation, and limited attenuation of vessels and para-arteriolar preservation of the RPE (PPRPE). Patients frequently have a Coats-like exudative vasculopathy. ${ }^{3,4}$

At present, no treatment is available for LCA or allied retinal dystrophies. However, several treatment strategies are under study, and some have reached the human clinical trial phase. ${ }^{15-19}$ Although all other potential treatments would also benefit from more profound knowledge of the underlying genetic defects and disease mechanisms, this is especially true for gene replacement therapy. Although the identification of 
the causal mutations in known LCA genes is technically not difficult (e.g., using sequence analysis), the genetic heterogeneity and complexity of several LCA genes has hampered their routine molecular analysis. For this purpose, high-throughput techniques are necessary. One such technique, allele-specific primer extension (APEX), has been used in conjunction with a genotyping microarray chip to detect mutations in the $A B C A 4$ gene. Mutations in this gene are associated with Stargardt disease, autosomal recessive cone-rod dystrophy, a panretinal autosomal recessive rod-cone dystrophy, and possibly agerelated macular degeneration. ${ }^{20-22}$ This chip has been a robust and cost-effective screening tool. ${ }^{23,24} \mathrm{~A}$ similar microarray chip was recently developed for genotyping 300 known mutations in six LCA genes. 5

In this study, we ascertained 58 unrelated patients with LCA, who predominantly live in Belgium and the Netherlands, and we identified the molecular causes in one third of the cases by means of the microarray chip. Clinical data of patients with mutations in the known LCA genes were analyzed retrospectively and all but three were re-examined for the purpose of this study to establish genotype-phenotype correlations.

\section{Methods}

\section{Patients}

DNA samples from 58 unrelated white patients with a clinical diagnosis of LCA were collected from Belgium (36 cases; Ghent University Hospital, University Hospital Leuven), The Netherlands (20 cases; Rotterdam Eye Hospital, Rotterdam; Sensis International, Grave; and Radboud University Nijmegen Medical Centre, Nijmegen), and Germany (2 cases; University Hospital Heidelberg and the University School of Medicine, Berlin). All procedures were approved by the ethics boards of the collaborating institutes and adhered to the tenets of the Declaration of Helsinki. All samples were acquired after written informed consent was obtained from the patient or in the case of children, their legal guardians.

LCA was defined as bilateral visual loss noticed before the age of 6 months, accompanied by wandering nystagmus and an undetectable or significantly reduced electroretinogram (ERG). Only patients who had not been genotyped previously were included in this study. Patients with a juvenile-onset RP (onset after 1 year of age) or a syndromic form of LCA, such as Joubert syndrome, were excluded.

\section{Microarray Mutation Analysis}

Standard protocols were used to extract DNA from peripheral blood leukocytes. ${ }^{25}$

The LCA microarray chip contained sense and antisense oligonucleotides (25-mers) corresponding to 301 previously identified diseaseassociated sequence variants in six of seven currently known LCA genes: AIPL1 (25 sequence variants), CRB1 (68 sequence variants), CRX (29 sequence variants), GUCY2D (66 sequence variants), RPE65 (81 sequence variants), and RPGRIP1 (32 sequence variants). ${ }^{26}$ In addition, the chip contained disease-associated sequence variants in two early-onset RP genes: LRAT (two sequence variants) and MERTK (three sequence variants).

In brief, 68 DNA fragments containing the 301 sequence variants were amplified, and PCR-products were visualized on agarose gels. The PCR products were pooled, denatured, and hybridized to the microarray chips. The sequence variants were detected by a sequence-specific primer extension using dideoxynucleotides. For technical details regarding the allele-specific primer extension (APEX) method, see Jaakson et al. ${ }^{23}$ The results obtained from the LCA microarray chip were confirmed through bidirectional sequencing of the relevant amplicons (ABI3730 and ABI3100 Genetic Analyzers; Applied Biosystems, Inc. [ABI], Foster City, CA). Automatic analysis was performed by a basecaller (ABI).
At the time of mutation chip analysis, RDH12 had not yet been identified. We therefore sequenced the seven protein coding exons of RDH12 in all Dutch and German patients. ${ }^{27}$

In those patients in whom one mutation was identified, the entire gene was sequenced. Primers and PCR conditions have been described. ${ }^{3,9,10,28}$ Four novel sequence changes were identified. One of them was a new GUCY2D (c.2837C $\rightarrow$ T; p.A946V) missense mutation that was subsequently tested in DNA from 93 anonymous healthy Dutch control individuals by restriction fragment length polymorphism (RFLP) analysis. BstUI cuts the normal but not the mutant PCR product containing exon 15 (288 bp) in 154- and 134-bp fragments. When parents or unaffected siblings were available, segregation analysis was performed.

\section{Phenotyping}

In patients with presumed pathologic sequence variants, clinical data were retrospectively collected. In most cases, follow-up clinical information was available, and we used the data from the first and last examinations. At the first visit, parents and patients were questioned about the age of onset of symptoms such as night blindness, photophobia, and photoattraction. A family history was taken and questions concerning pregnancy, birth, birth defects, and consanguinity were asked. Pupillary reactions were observed, and nystagmus and photophobia were evaluated. Ophthalmic examination included best-corrected, age-appropriate visual acuity testing and measurement of objective refractive error after cycloplegia. The anterior segment was examined by slit lamp biomicroscopy followed by funduscopy after pupillary dilation. Goldmann visual field results were obtained when possible. ERGs made according to pediatric protocols were obtained in all patients but three at the collaborating institutes. Color vision was tested in four patients with a low-vision edition of the Panel D-15 test (large surfaces) under bright daylight illumination (3500 lux; color temperature of approximately $4500 \mathrm{~K}$ ), higher than levels normally used in color vision testing (1600-1900 lux; color temperature of 6500 K). ${ }^{29}$ Images of the fundus were acquired with a fundus photography system (Topcon, Tokyo, Japan). Interpretable autofluorescence images were obtained in two patients with the same system. Interpretable autofluorescence and red-free images were acquired with the Heidelberg Retinal Angiograph (HRA2; Heidelberg Engineering, Heidelberg, Germany) in one patient.

\section{Results}

\section{Genotypes}

The molecular genetic results are summarized in Table 1 . The microarray chip allowed the identification of homozygous or compound heterozygous sequence variants in 14 of 58 patients and single heterozygous sequence variants in 6 of 58 . All variants were confirmed by using sequence analysis of the relevant exons. In the six heterozygous patients, the proteinencoding exons of the respective genes were sequenced, and four novel sequence variants were identified.

\section{Phenotypes}

All available clinical data of the first visit as well as funduscopic findings at the last examination are summarized in Table 2 . In the following sections, a summary of relevant clinical information is provided. All patients had an ERG performed before the age of 12 months, which was nonrecordable in all except patients 21394, 24105, and 246V1, in whom the ERG was severely reduced.

\section{AIPL1}

Genotypes. All three patients with mutations in AIPL1 were homozygous for p.R278W. 
TABLE 1. Mutations Identified in Patients with LCA and Results from Segregation Analysis

\begin{tabular}{|c|c|c|c|c|c|c|c|c|}
\hline \multirow[b]{2}{*}{ Gene } & \multirow[b]{2}{*}{ Patient } & \multicolumn{3}{|c|}{ Variant 1} & \multicolumn{3}{|c|}{ Variant 2} & \multirow{2}{*}{$\begin{array}{c}\begin{array}{c}\text { Segregation } \\
\text { of }\end{array} \\
\text { Mutations }\end{array}$} \\
\hline & & Exon & Nucl. Change & AA Change & Exon & Nucl. Change & AA Change & \\
\hline \multirow[t]{3}{*}{ AIPL1 } & 14962 & 6 & c. $834 \mathrm{G} \rightarrow \mathrm{A}$ & p.W278X & 6 & c. $834 \mathrm{G} \rightarrow \mathrm{A}$ & p.W278X & NT \\
\hline & $20143^{*}$ & 6 & c. $834 \mathrm{G} \rightarrow \mathrm{A}$ & p.W278X & 6 & c. $834 \mathrm{G} \rightarrow \mathrm{A}$ & p.W278X & Yes \\
\hline & 2040V1 & 6 & c. $834 \mathrm{G} \rightarrow \mathrm{A}$ & p.W278X & 6 & c. $834 \mathrm{G} \rightarrow \mathrm{A}$ & p.W278X & Yes \\
\hline \multirow[t]{9}{*}{$C R B 1$} & 21394 & 9 & c. $2843 \mathrm{G} \rightarrow \mathrm{A}$ & p.C948Y & 9 & c. $3664 \mathrm{C} \rightarrow \mathrm{T}$ & p.Q1222X & Yes \\
\hline & 21405 & 9 & c. $2843 \mathrm{G} \rightarrow \mathrm{A}$ & p.C948Y & 7 & c. $2234 \mathrm{C} \rightarrow \mathrm{T}$ & p.T745M & Yes \\
\hline & 21551 & 9 & c. $2843 \mathrm{G} \rightarrow \mathrm{A}$ & p.C948Y & 8 & c. $2842+5 \mathrm{G} \rightarrow \mathrm{A}$ & splice defect & Yes \\
\hline & 21554 & 9 & c. $2843 \mathrm{G} \rightarrow \mathrm{A}$ & p.C948Y & 8 & c. $2842+5 \mathrm{G} \rightarrow \mathrm{A}$ & splice defect & Yes \\
\hline & 21669 & 9 & c. $2843 \mathrm{G} \rightarrow \mathrm{A}$ & p.C948Y & 7 & c. $2234 \mathrm{C} \rightarrow \mathrm{T}$ & p.T745M & Yes \\
\hline & 246V1 & 7 & c. $2401 \mathrm{~A} \rightarrow \mathrm{T}$ & p.K801X & 5 & c. $1084 \mathrm{C} \rightarrow \mathrm{T}$ & p.Q362X & NT \\
\hline & 278V1 & 7 & c. $2401 \mathrm{~A} \rightarrow \mathrm{T}$ & p.K801X & 8 & c. $2688 \mathrm{~T} \rightarrow \mathrm{A}$ & p.C896X & NT \\
\hline & 2148V1 & 7 & c. $2401 \mathrm{~A} \rightarrow \mathrm{T}$ & p.K801X & 8 & c. $2688 \mathrm{~T} \rightarrow \mathrm{A}$ & p.C896X & Yes \\
\hline & $3460 \mathrm{~V} 1$ & 7 & c. $2401 \mathrm{~A} \rightarrow \mathrm{T}$ & p.K801X & - & - & - & NA \\
\hline \multirow[t]{6}{*}{$G U C Y 2 D$} & 441 & 8 & c. $1694 \mathrm{~T} \rightarrow \mathrm{C}$ & p.F565s & 12 & c. $2302 \mathrm{C} \rightarrow \mathrm{T}$ & p.R768W & Yes \\
\hline & 20955 & 12 & c. $2302 \mathrm{C} \rightarrow \mathrm{T}$ & p.R768W & 12 & c. $2302 \mathrm{C} \rightarrow \mathrm{T}$ & p.R768W & Yes \\
\hline & $21067^{*}$ & 12 & c. $2302 \mathrm{C} \rightarrow \mathrm{T}$ & p.R768W & 15 & c. $2837 \mathrm{C} \rightarrow \mathbf{T}$ & p.A946V & Yes \\
\hline & 21557 & 12 & c. $2302 \mathrm{C} \rightarrow \mathrm{T}$ & p.R768W & 12 & c. $2302 \mathrm{C} \rightarrow \mathrm{T}$ & p.R768W & Yes \\
\hline & 22018 & 12 & c. $2302 \mathrm{C} \rightarrow \mathrm{T}$ & p.R768W & 12 & c. $2302 \mathrm{C} \rightarrow \mathrm{T}$ & p.R768W & NT \\
\hline & $22597^{*}$ & 8 & c. $1694 \mathrm{~T} \rightarrow \mathrm{C}$ & p.F565S & 8 & c. $1694 \mathrm{~T} \rightarrow \mathrm{C}$ & p.F565s & NT \\
\hline RPE65 & 23207 & 5 & c. $370 \mathrm{C} \rightarrow \mathrm{T}$ & p.R124X & 14 & c.1590delC & p.F530fs & Yes \\
\hline
\end{tabular}

Bold sequence variants: novel pathogenic mutations identified by sequence analysis. —, no second allele found; AA, amino acid; NA, not applicable; Nucl., nucleotide; NT, not tested.

* Consanguineous parents.

Phenotypes. Over a 15-year period, the visual acuity of patient 14962 remained stable. When he was initially examined during his first year of life because of nystagmus, funduscopy was normal. At the age of 15 , a bull's eye maculopathy as well as increased pigment spicules in the periphery were observed.

Patient 20143 was first examined after he failed to fix and follow objects at the age of 3 months. He was mentally handicapped, and he regularly poked his eyes (oculodigital sign) and had weak and slow pupillary reactions. At the age of 7.5 years, visual acuity (VA) was 0.003 (OU) and the lenses showed a nuclear opacity. Funduscopy revealed a pale optic disc and attenuation of the retinal vasculature, with neither obvious maculopathy nor peripheral intraretinal pigmentation.

When patient 2040V1 was first seen at the age of 5 months because of nystagmus, funduscopy revealed diffuse alterations of the retinal pigment epithelium and mild attenuation of vessels. She was night-blind and preferred a well-lit environment. At 8 years of age, she displayed nystagmus with a predominant slow horizontal component. Best-corrected visual acuity was 0.02 with a refraction of $+7.5 \mathrm{OU}$. Axial length was $19.6 \mathrm{~mm}$ OD and $19.9 \mathrm{~mm}$ OS. On funduscopy, a bull's eye maculopathy was present with diffuse RPE alterations with only limited spicular intraretinal pigmentation (Fig. 1a). Sub- or deep intraretinal hyperfluorescent spots were seen on autofluorescence imaging (Fig. 1b).

\section{CRB1}

Genotypes. Eight of nine patients carrying mutations in CRB1 were compound heterozygous. The p.C984Y and p.K801X alleles were found in five and four patients, respectively. Two patients with CRB1 mutations were compound heterozygous for mutations p.C948Y and c.2842+5G $\rightarrow \mathrm{A}$, and one of them (patient 21551) also carried the p.V96I AIPL1 sequence variant. The latter sequence change was not considered to be pathologic, because it was identified in heterozygous form in 11 of 186 healthy control subjects. Two new nonsense mutations were identified in CRB1: p.Q362X and p.Q1222X.
Phenotypes. Patient 21394 first presented at the age of 3 months with torticollis presumably related to nystagmus. His fundus was then normal. At the age of 5 years, his VA had decreased slightly from 0.05 to 0.02 OU. On funduscopy, he had several characteristics of RP12, including pink optic discs, relatively normal arterioles, and extensive RPE atrophy, both in the posterior pole and in the periphery, with nummular pigmentations. He lacked PPRPE.

Patient 21405 was first examined in the first year of life because of night blindness. At $0.17 \mathrm{OD}$ and $0.20 \mathrm{OS}$, her visual acuity was then better than any other patient in this entire study cohort. Funduscopy showed macular and peripheral mild RPE changes as the only abnormalities. At age 14, the typical RP12 phenotype was seen with macular and optic disc edema, a dysplastic macular region, perivascular sheathing, mild arteriolar attenuation, and PPRPE (Fig. 1i). Goldmann perimetry performed at the time showed an absolute ring scotoma with relatively well preserved central fields $\left(50^{\circ} \mathrm{di}-\right.$ ameter OD, $\left.30^{\circ} \mathrm{OS}\right)$.

The first symptom noticed in patient 21551 was a failure to develop eye contact at age 4 months. When the patient was initially seen at that age, ERG responses were absent. Very limited clinical data were available, but at 13 years of age, he had very poor pupillary reactions with a residual visual acuity and stable hypermetropic refraction. Goldmann visual field testing showed preservation of the central $15^{\circ}$ field, albeit with a considerable decrease in sensitivity.

LCA was diagnosed in patient 21554 at 2 months of age after the oculodigital sign had been noticed and ERG responses were absent. When examined at age 3 , she preferred a well-lit environment and was reported unable to see in dim light. She had a predominantly horizontal slow nystagmus and esotropia of the right eye. The VA was virtually stable when she was examined at the age of 12 . She had by then lost nearly all ability to recognize basic colors. A small posterior lenticular opacity was present inferior to the visual axis. On funduscopy she showed the RP12 phenotype and over a 9-year period both the aspect and size of the dysplastic regions in both maculae 


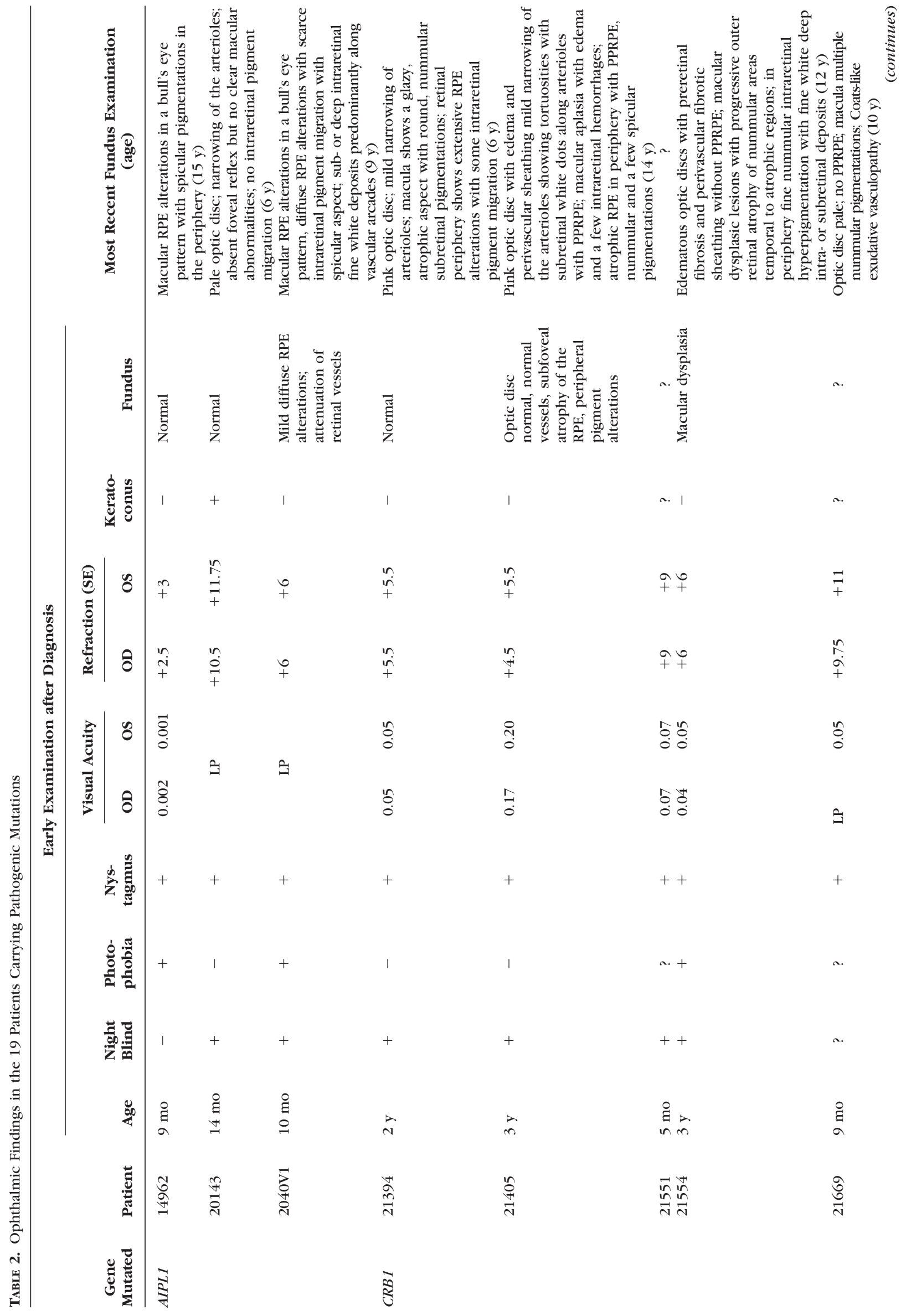




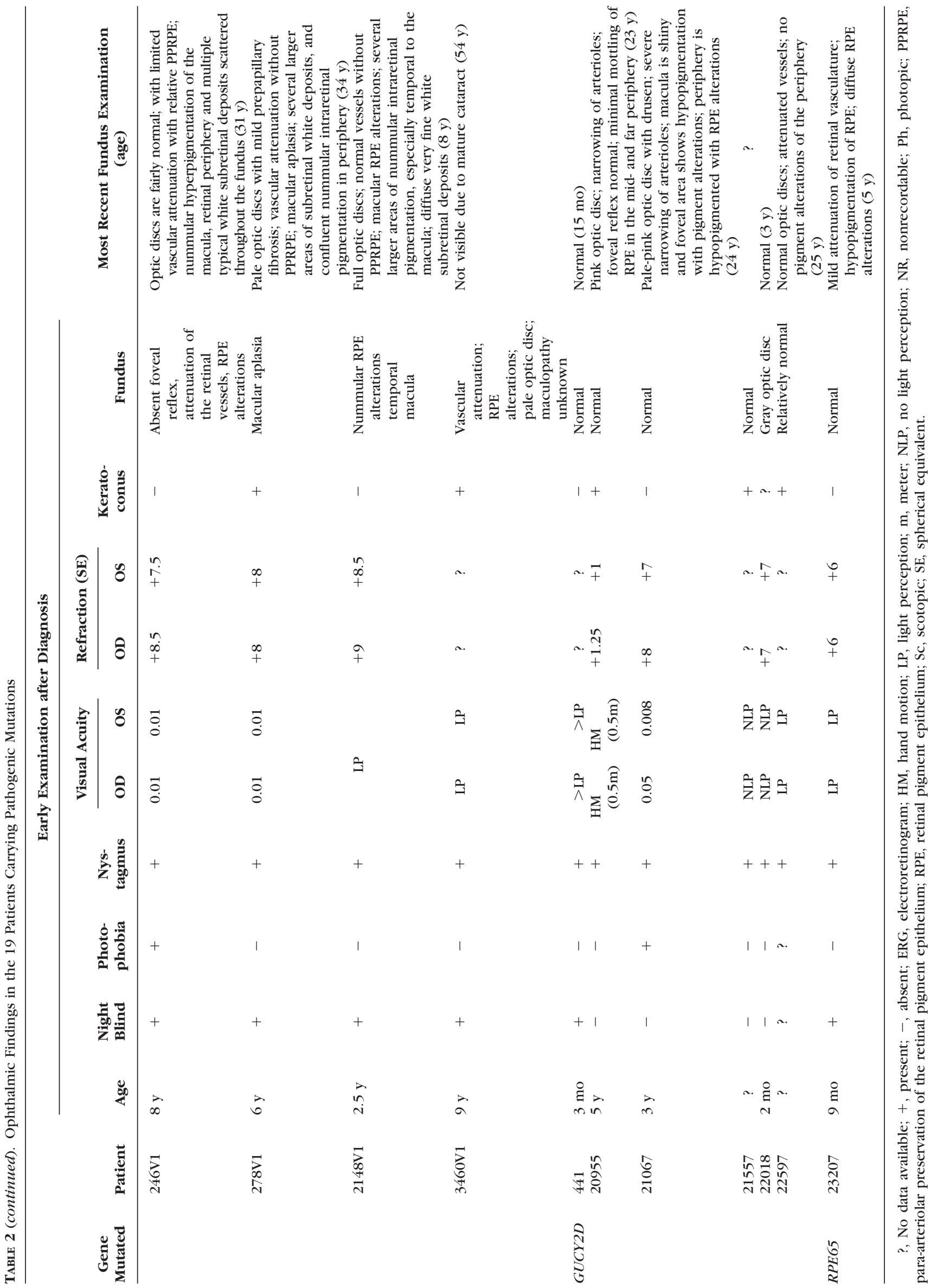




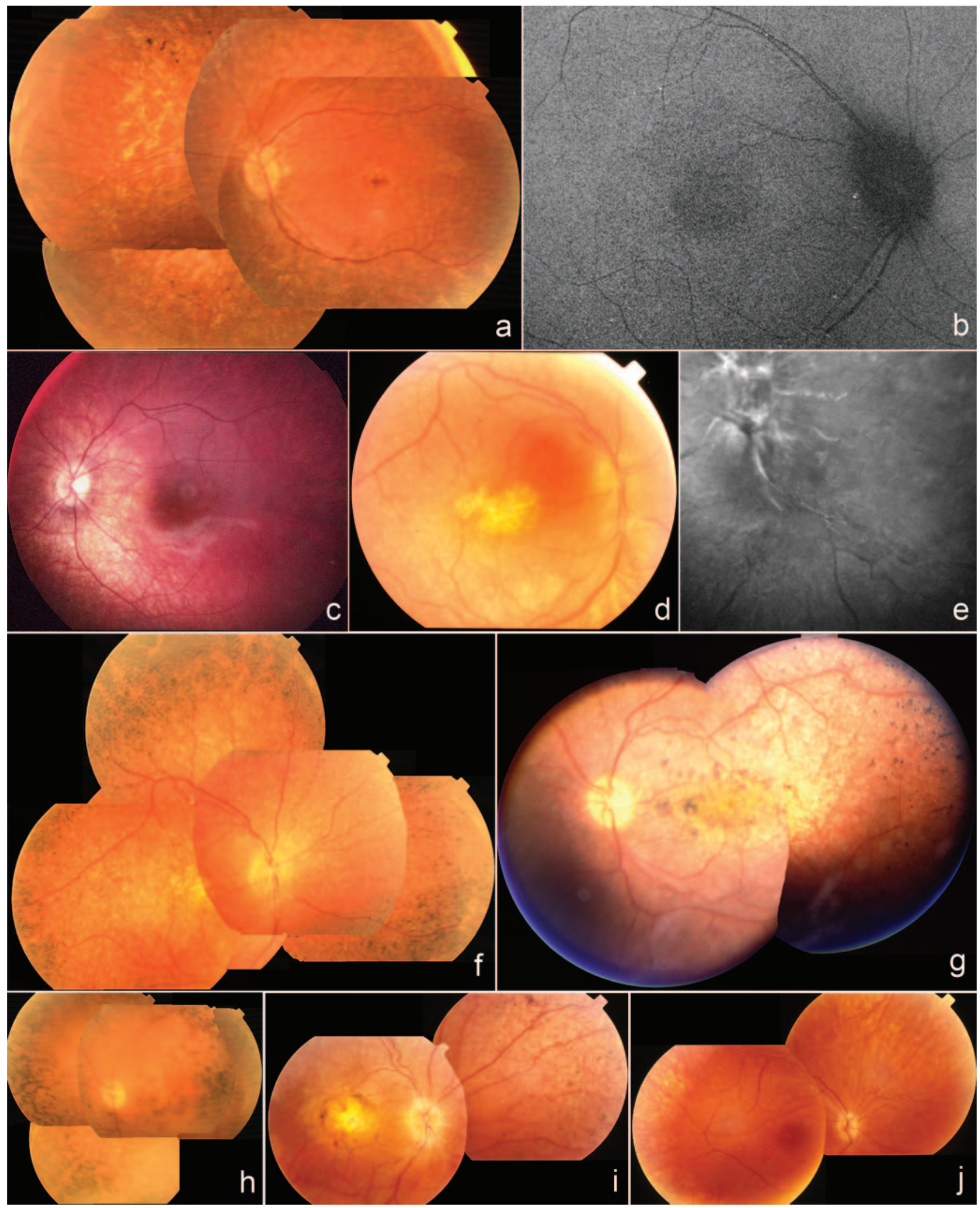

Figure 1. Fundus photographs of patients with LCA. (a) Patient 2040V1 (AIPL1 p.W278X/p.W278X) at age 9: composite of fundus of the left eye (OS), showing macular dystrophy in a bull's eye pattern, diffuse RPE alterations with scarce intraretinal pigment migration with spicular aspect, and sub- or deep intraretinal fine white deposits predominantly along vascular arcades. (b) Patient 2040V1 (AIPL1 p.W278X/p.W278X) at age 9: composite of autofluorescence image of fundus of right eye (OD), showing presence of sub- or deep intraretinal fine white deposits predominantly along vascular arcades that hyperfluoresce. (c) Patient 23207 (RPE65 p.R124X/p.F530fs) at age 6.5 years: a relatively hypopigmented fundus of OS 
remained virtually stable (Figs. 1d, 1f). Perivascular sheathing of retinal vessels around the optic disc was better seen on red-free imaging (Fig. 1e).

Patient 21669 was born with palatoschisis. The disease was diagnosed when he was 6 months of age, when strabismus was noted and ERG responses were absent. When seen last at age 10 years, he showed funduscopic characteristics of RP12 with Coats-like vasculopathy in both eyes. PPRPE was not present.

Patient 246V1 had received a diagnosis at age 4 months after presenting with nystagmus. Over 30 years, his VA of 0.01 OU and hypermetropia with pronounced astigmatism of +5.0 $\mathrm{D}(-5.5 \mathrm{D}) 30^{\circ} \mathrm{OD}$ and $+4.5 \mathrm{D}(-5.25 \mathrm{D}) 140^{\circ}$ OS has remained fairly stable. When last seen at age 30 , he preferred bright light. His nystagmus was horizontal. A subcapsular cataract was present in both eyes (OS $>$ OD). Despite the considerable astigmatism, there were no signs of keratoconus. Over a period of 16 years, some of the funduscopically visible RP12 characteristics, such as nummular hyperpigmentation and subretinal deposits, increased (Figs. 1g, 1h). He had basic color vision as tested on a low-vision Panel D-15 test, when ambient light was sufficiently intense.

In patient $278 \mathrm{~V} 1$ the oculodigital sign had been noted since childhood. At the age of 34 years, her VA had decreased slightly to 0.005 OU due to scarring after acute hydrops of her bilateral keratoconus. Her vision was better in bright light. She had pronounced enophthalmos, esotropia, and nystagmus, which also had a predominant horizontal component. On funduscopy, a steady increase in the white subretinal deposits and nummular pigmentation was noted over the years. The size of the macular aplastic lesions had not changed since birth.

A diagnosis of LCA was made in patient $2148 \mathrm{~V} 1$ at age 4 months because of a failure to fix and follow objects. ERG responses were absent. At the age of 8 years, he preferred bright light and had a VA of only hand movements. He had enophthalmos due to frequent eye-poking, and he displayed horizontal nystagmus. Slit lamp examination findings stayed unremarkable over the years. The nummular intraretinal pigmentation and number of deposits increased. There was some perception of colors, as measured with a modified Panel D-15 test.

Patient 3460V1 was a 55-year-old blind woman who was severely mentally retarded due to meningitis in the first weeks of life. When LCA was diagnosed at age 3 months she was failing to fix and follow objects, and ERG responses were absent. She frequently poked her eyes. After several painful episodes of acute corneal hydrops consequent from keratoconus, her right eye was enucleated at age 53. Because of the presence of a dense cataract, funduscopy of the left eye was impossible, and, because of mental retardation, visual acuity was unmeasurable.

\section{CRX}

In patient 22654, a heterozygous sequence change (p.Y142C) in the $C R X$ gene was identified. Sequencing of the entire gene confirmed the presence of this variant but did not reveal a second change. Segregation analysis of the unaffected consanguineous parents revealed the mother to be homozygous for this sequence change and the father to carry two wild-type alleles. To rule out the possibility that we failed to amplify a wild-type allele by PCR in the mother due to a polymorphic sequence change underneath one of the amplicon primers, we repeated the sequence analysis with different primers and confirmed the homozygosity of the p.Y142C variant in the DNA of the patients' mother. The maternal grandparents had normal vision. The sequence change was therefore considered a benign variant. Therefore, we conclude that 33 of 116 disease alleles were identified by chip analysis alone.

\section{GUCY2D}

Genotypes. The p.R768W mutation represented 8 of 12 alleles in five of six patients with GUCY2D-related LCA. One of the compound heterozygotes carried a novel mutation, p.A946V, which was absent in 93 control individuals.

Phenotypes. LCA was diagnosed in patient 441 at the age of 3 months after she failed to fix and follow objects. When examined at the age of 1 year, she frequently poked her eyes. She was able to follow objects, provided she was in a well-lit environment, after having started staring at lights at the age of 5 months. Her fundus examination had been unremarkable since birth.

Patient 20955 received a diagnosis of LCA in the weeks after birth because of eye poking, which she continued until the age of 8 years. ERG responses were absent. At the age of 23 she preferred a dim environment. Her fundus was fairly normal, apart from arteriolar attenuation.

After a diagnosis because of strabismus at 6 months of age, patient 21067 was photophobic as a child, which markedly decreased later in life, and at the age of 22 she instead preferred bright light. She had esotropia of the left eye. Apart from arteriolar attenuation and mild RPE alterations in the fovea, the fundus examination was unremarkable (Fig. 1j).

LCA was diagnosed in patient 21557 at the age of 4 months, because he failed to fix and follow objects. His ERG responses were absent, and he was completely blind. At the age of 39, he had a divergent strabismus as well as bilateral dense cataracts that precluded funduscopy.

A diagnosis was made in patient 22018 at the age of 2 months after he failed to fix and follow objects. He showed the oculodigital sign and episodic head turns. At the age of 2.5 years, there was no perception of light, and his pupils were

with mild attenuation of arterioles and diffuse RPE alterations. (d) Patient 21554 (CRB1 p.C948Y/c.2842+5G $\rightarrow$ A): posterior pole of OD at age 7 showing edematous optic disc with preretinal fibrosis, perivascular sheathing, macular dysplasia, and fine, white, deep intra- or subretinal deposits. (e) Patient 21554 (CRB1 p.C948Y/c.2842+5G $\rightarrow$ A): red-free image of optic disc and inferotemporal peripapillary area of OS at age 12 showing a better visualization of perivascular fibrotic sheathing. (f) Patient 21554 (CRB1 p.C948Y/c.2842+5G $\rightarrow$ A): composite of fundus of OD at age 12 showing edematous optic disc with preretinal fibrosis and perivascular fibrotic sheathing without clear PPRPE apart from vessel inferonasal to OD; macular dysplasia remains stable, whereas there is fine nummular intraretinal hyperpigmentation with fine, white, deep intra- or subretinal deposits in the mid- and far periphery. (g) Patient 246V1 (CRB1 p.K801X/p.Q362X): composite fundus picture of OS at age 14: Note that optic disc is fairly normal; the macula is dysplastic; limited vascular attenuation with relative PPRPE; nummular hyperpigmentation in the macula and the retinal periphery and multiple white subretinal deposits scattered throughout the fundus. (h) Patient 246V1 (CRB1 p.K801X/p.Q362X): composite fundus picture of OS at age 30 shows limited visibility due to subcapsular cataract, stable dysplasia of the macula but a dramatic increase in the number of nummular pigmentations in the posterior pole, vascular attenuation, mixed nummular and spicular hyperpigmentation in the retinal periphery, and multiple white subretinal deposits scattered throughout the fundus. (i) Patient 21405 (CRB1 p.C948Y/p.T745M) at age 14: composite of fundus of OD showing edematous optic disc with perivascular sheathing, mild narrowing of the arterioles showing tortuosities with subretinal white dots along arterioles with PPRPE, macular aplasia, atrophic RPE in the periphery with PPRPE, and nummular and a few spicular pigmentations. The posterior pole showed edema with a few intraretinal hemorrhages. (j) Patient 21067 (GUCY2D p.R768W/p.A946V): composite of fundus of OD at age 22 showing arteriolar attenuation and mild RPE alterations in the fovea. 
wide and responded only minimally to bright light. Funduscopy was normal.

Patient 22597 belonged to a family with 10 siblings, three of which had LCA. Therefore, an early clinical diagnosis was made soon after birth. There was congenital nystagmus, and her vision had never been better than light perception. Recently, she had acute hydrops of a keratoconus in one eye. When she was 25 years of age, attenuation of the retinal vasculature was the only abnormality noted on funduscopy.

\section{RPE65}

Genotype. In this cohort, one patient had LCA due to mutations in RPE65. The p.R124X mutation was identified by the chip analysis, and a novel frameshift mutation (p.F530fs) was identified by sequence analysis.

Phenotype. Patient 23207 did not make eye contact at 2 months and had night blindness at the age of 6 years. He had a very poor VA in the first year of life, with gradual improvement up to $0.16 \mathrm{OD}$ and $0.12 \mathrm{OS}$, with a refraction of $-2.5 \mathrm{OD}$ and -3.5 OS at the age of 6 years. Nystagmus was present. He had a visual field of $60^{\circ}$ and perceived the main colors. His fundus was relatively hypopigmented, with mild attenuation of the arterioles and diffuse RPE alterations (Fig. 1c).

\section{Discussion}

The mutation-screening protocol applied in this study consisted of an initial microarray-based mutation analysis, followed by sequence analysis of genes in those patients with LCA in whom single heterozygous mutations were identified. In addition, mutations in patients who were either homozygous or compound heterozygous were confirmed by sequence analysis.

This approach allowed the identification of 37 disease alleles in 19 (33\%) of 58 patients with LCA. These represent nine different previously identified and four novel sequence variants. We identified one heterozygous pathologic LCA variant in patient $3460 \mathrm{~V} 1$ among 58 patients with LCA. Possibly, mutations outside the protein coding exons and their immediate vicinity were missed, as are all heterozygous deletions. Also, the heterozygous variant in $3460 \mathrm{~V} 1$ could be unrelated to LCA, since the aggregate heterozygosity frequency of mutations in the AIPL1, CRB 1, CRX, GUCY2D, RPE65, and RPGRIP1 genes in the general population is $1.3 \%$, assuming an LCA incidence of 1 in 100,000 (Cremers FPM, unpublished data, 2005).

In 13 families, segregation analysis of the sequence variants was compatible with autosomal recessive inheritance. In five other families in whom no segregation analysis could be performed, three patients apparently were homozygous for a disease-causing sequence change. However, the presence of heterozygous deletions cannot be ruled out in these cases.

Mutations in this patient cohort were most frequently identified in CRB1 (nine cases, $15.5 \%$ ), followed by GUCY2D (six cases, 10.3\%), AIPL1 (three cases, 5.2\%), and RPE65 (one case, 1.7\%). No pathologic mutations were found in $C R X, R D H 12$ (in 22 patients), and RPGRIP1. The infrequent involvement of $C R X, R D H 12$, and RPGRIP1 in LCA is in accordance with the results of previous studies. ${ }^{6,8,27,30}$ In addition, it is noteworthy that RPE65 seems to account for only a minority of cases $(1.7 \%)$ in comparison to reported frequencies in other studies of 3\% to $15 \% .{ }^{31-34}$ This variation may be due to the relatively small patient cohort, the variable mutation detection strategies and the variable inclusion criteria applied by different groups. In general, mutations in the RPE65 gene are more frequently observed in patients with early-onset RP rather than LCA. ${ }^{35}$ (Yzer S, unpublished results, 2005).
All three patients carrying AIPL1 mutations were homozygous for the p.W278X allele. Previously, nine patients from different ethnic backgrounds were described to be homozygous for this change by Dharmaraj et al. ${ }^{36}$ The phenotype of these patients consisted of severe night blindness, some degree of maculopathy, attenuated vessels, and spicular intraretinal pigment migration in the periphery after several years of evolution. Keratoconus was observed in 6 of 19 patients with distinct AIPL1 mutations. The patients described in this study showed an apparently normal fundus on initial examination, which progressively evolved into peripheral spicular intraretinal pigment migration in early childhood. High hyperopia and a very poor visual acuity appear to be consistent findings. Night blindness is a frequent, albeit variable, characteristic. ${ }^{6,36}$

Eight of nine patients with CRB1 mutations showed either all or some funduscopic characteristics of the RP12 phenotype, including relatively normal optic discs and vessels, a distinct maculopathy, and a nummular rather than a spicular type of pigmentation. Yellow-white retinal dots were present in eight patients and PPRPE in three. Findings less frequently observed in RP12 or patients with CRB1 mutations, such as Coats-like exudative vasculopathy, vascular sheathing, optic disc edema, macula dysplasia, and keratoconus were also observed in our patients. Moderate to high hyperopia and nystagmus are also associated with CRB1-associated LCA. ${ }^{4,6,8,37}$ This RP12 phenotype is easily distinguishable from other RP-like phenotypes.

The $C R X$ p.Y142C variant has been identified in heterozygous form in a patient with autosomal dominant LCA (Kaplan $\mathrm{J}$, personal communication, 2004). In our cohort, one patient from a consanguineous marriage was a heterozygous carrier of this variant. Sequence analysis revealed the healthy mother to be homozygous for this sequence change, suggesting that the p.Y142C change is nonpathogenic. Because the maternal grandparents had normal vision, a metabolic interference model in which homozygous mutations abolish each other's pathologic effects, seems unlikely.

Mutations in the GUCY2D gene in LCA were first reported in Mediterranean families. ${ }^{10}$ In the LCA cohort of this study, the p.R768W mutation was found in eight of 12 (67\%) alleles. Four of the five patients carrying this allele were of Belgian or Dutch origin, and the fifth was of Belgian/Moroccan descent. Segregation analysis showed that the p.R768W allele came from her Belgian mother. Hanein et al. ${ }^{6}$ found the p.R768W allele in only 1 of 75 GUCY2D alleles in 38 patients, most of which were from Mediterranean countries. This difference in frequency of the p.R768W mutation may be suggestive of a founder effect in the northwestern region of Europe. The three patients with the homozygous p.R768W GUCY2D mutation are severely affected, did not show light perception at initial examination, and had no signs of improvement later in life. In most patients, the initial fundus was normal which, later in life, showed attenuated vasculature and subtle pigmentations. In accordance with previous findings by Dharmaraj et al., ${ }^{30}$ refraction in GUCY2D-related patients with LCA varies from mild to severe hyperopia with VA showing a spectrum from no light perception to 0.1 . In contrast, Hanein et al. ${ }^{6}$ generally found hypermetropia $(>+7)$.

A novel frameshift mutation, p.F530fs, was identified in the RPE65 gene in a patient heterozygous for p.R124X. The phenotype of this patient is similar to previously published phenotypes with very poor visual acuity early in life that gradually improves, myopia, and some residual visual field function. Overall, the phenotype of patients with LCA with RPE65 mutations seems relatively mild in comparison to other forms of LCA. $^{6,34,35}$

Our patient cohort was relatively small, but our clinical data suggest that patients with homozygous p.W278X AIPL1 mutations have a very poor visual acuity, with hyperopia and an 
initially normal fundus, which progresses to an RP-like fundus in early childhood. In general, GUCY2D-related LCA results in the most severe visual impairment with a relatively normal fundus in the mid-20s. Furthermore, this study supports the existence of a fairly typical and consistent phenotype of CRB1associated LCA, including a maculopathy ranging from macular RPE alterations to macular aplasia, fine white sub- or deep intraretinal drusen-like deposits, and nummular rather than spicular hyperpigmentation in both the macula and periphery. The phenotypical data also confirm that PPRPE is a rather inconsistent feature in patients with CRB1 mutations, probably due to its transient nature.

In this study, $C R B 1$ was the most frequently involved gene (9/19 patients) in a cohort of predominantly Belgian and Dutch patients. In a Spanish LCA cohort, mutations in CRB1 turned out to underlie two thirds of all cases with mutations in the known LCA genes (Vallespin E, personal communication, 2005). In contrast, no CRB1 mutations were detected in a Canadian cohort of 24 patients with LCA, suggestive of sizeable geographic differences in the occurrence of mutations. ${ }^{38}$

Taken together, the clinical findings in our patients suggest that both photophobia and night blindness do not seem to be reliable clinical features that can be used to direct gene analysis, as was proposed by Hanein et al. ${ }^{6}$ Indeed, within the AIPL1-, GUCY2D-, and CRB1-associated patient cohorts in our study, both photophobia and night blindness were noted.

A comprehensive mutation analysis study of all currently known LCA genes has been performed. ${ }^{6}$ Excluding this study's findings in the TULP1 gene, as the TULP1 gene was not analyzed in our study, mutations were identified in $44.4 \%$ of the disease alleles by using a combination of linkage analysis of known genes, denaturing high-pressure liquid chromatography mutation scanning, and direct sequencing. Our approach (i.e., a first-pass mutation detection of known mutations with the LCA microarray chip followed by sequence analysis of patients with heterozygous mutations) allowed the detection of $31.9 \%$ (37/116) of disease alleles. Hence, approximately $72 \%$ (31.9/ 44.4) of the expected disease alleles were identified with a relatively small effort. We therefore believe that the LCA mutation chip offers a cheap and efficient first-pass screening test. This robust technique is generally applicable and allows more carefully directed efforts of subsequent standard sequence analysis. Furthermore it is independent of a detailed clinical history necessary for a decisional molecular diagnostic flowchart, as outlined by Hanein et al. ${ }^{6}$ Finally, by regular addition of newly identified alleles, the efficiency of the microarray will continuously improve.

\section{Acknowledgments}

The authors thank the patients who kindly agreed to take part in the study and Diana Cremers, Bellinda van den Helm, Sarah De Jaegere, Cees van Oostende, Bert Smit, and Saskia D. van der Velde-Visser for expert technical assistance.

\section{References}

1. Leber T. Ueber Retinitis pigmentosa und angeborene Amaurose. Albrecht von Grafes Arch Ophthalmol. 1869;15:1-25.

2. Waardenburg PJ, Schappert-Kimmijser J. On various recessive biotypes of Leber's congenital amaurosis. Acta Ophthalmol. 1963;41: 317-320.

3. den Hollander AI, Ten Brink JB, De Kok YJM, et al. Mutations in a human homologue of Drosophila crumbs cause retinitis pigmentosa (RP12). Nat Genet. 1999;23:217-221.

4. den Hollander AI, Heckenlively JR, van den Born LI, et al. Leber congenital amaurosis and retinitis pigmentosa with Coats-like exudative vasculopathy are associated with mutations in the crumbs homologue 1 (CRB1) gene. Am J Hum Genet. 2001;69:198-203.
5. Dryja TP, Adams SM, Grimsby JL, et al. Null RPGRIP1 alleles in patients with Leber congenital amaurosis. Am J Hum Genet. 2001; 68:1295-1298.

6. Hanein S, Perrault I, Gerber S, et al. Leber congenital amaurosis: comprehensive survey of the genetic heterogeneity, refinement of the clinical definition, and genotype-phenotype correlations as a strategy for molecular diagnosis. Hum Mutat. 2004;23: 306-317.

7. Janecke AR, Thompson DA, Utermann G, et al. Mutations in RDH12 encoding a photoreceptor cell retinol dehydrogenase cause childhood-onset severe retinal dystrophy. Nat Genet. 2004; $36: 850-854$.

8. Lotery AJ, Jacobson SG, Fishman GA, et al. Mutations in the CRB1 gene cause Leber congenital amaurosis. Arch Ophthalmol. 2001; 119:415- 420.

9. Marlhens F, Bareil C, Griffoin JM, et al. Mutations in RPE65 cause Leber's congenital amaurosis. Nat Genet. 1997;17:139-141.

10. Perrault I, Rozet JM, Calvas P, et al. Retinal-specific guanylate cyclase gene mutations in Leber's congenital amaurosis. Nat Genet. 1996;14:461-464.

11. Sohocki MM, Sullivan LS, Mintz-Hittner HA, et al. A range of clinical phenotypes associated with mutations in CRX, a photoreceptor transcription-factor gene. Am J Hum Genet. 1998;63:1307-1315.

12. Sohocki MM, Bowne SJ, Sullivan LS, et al. Mutations in a new photoreceptor-pineal gene on $17 \mathrm{p}$ cause Leber congenital amaurosis. Nat Genet. 2000;24:79-83.

13. Cremers FPM, van den Hurk JAJM, den Hollander AI. Molecular genetics of Leber congenital amaurosis. Hum Mol Genet. 2002;11: 1169-1176.

14. McKay GJ, Clarke S, Davis JA, Simpson DAC, Silvestri G. Pigmented paravenous chorioretinal atrophy is associated with a mutation within the crumbs homolog 1 (CRB1) gene. Invest Ophthalmol Vis Sci. 2005;46:322-328.

15. Acland GM, Aguirre GD, Ray J, et al. Gene therapy restores vision in a canine model of childhood blindness. Nat Genet. 2001;28:9295.

16. Bennett J. Gene therapy for Leber congenital amaurosis. In: Bock G, Chader G, Goode J, eds. Retinal Dystrophies: Functional Genomics to Gene Therapy: Novartis Foundation Symposium 255. Chichester, UK: John Wiley \& Sons, Ltd.; 2004;195-202.

17. Chow AY, Chow VY, Packo KH, Pollack JS, Peyman GA, Schuchard R. The artificial silicon retina microchip for the treatment of vision loss from retinitis pigmentosa. Arch Ophthalmol. 2004;122:460 469.

18. Lai CM, Yu MJ, Brankov M, et al. Recombinant adeno-associated virus type 2-mediated gene delivery into the Rpe65-/- knockout mouse eye results in limited rescue (on-line). Genet Vaccines Ther. 2004; $2: 3$.

19. Tao W, Wen R, Goddard MB, et al. Encapsulated cell-based delivery of CNTF reduces photoreceptor degeneration in animal models of retinitis pigmentosa. Invest Ophthalmol Vis Sci. 2002;43:32923298.

20. Allikmets R, Shroyer NF, Singh N, et al. Mutation of the Stargardt disease gene (ABCR) in age-related macular degeneration. Science. 1997;277:1805-1807.

21. Allikmets R, Singh N, Sun H, et al. A photoreceptor cell-specific ATP-binding transporter gene (ABCR) is mutated in recessive Stargardt macular dystrophy. Nat Genet. 1997;15:236-246.

22. Gerber S, Rozet JM, van de Pol TJR, et al. Complete exon-intron structure of the retina-specific ATP binding transporter gene (ABCR) allows the identification of novel mutations underlying Stargardt disease. Genomics. 1998;48:139-142.

23. Jaakson K, Zernant J, Kulm M, et al. Genotyping microarray (gene chip) for the ABCR (ABCA4) gene. Hum Mutat. 2003;22:395- 403.

24. Klevering BJ, Yzer S, Rohrschneider K, et al. Microarray-based mutation analysis of the ABCA4 (ABCR) gene in autosomal recessive cone-rod dystrophy and retinitis pigmentosa. Eur J Hum Genet. 2004;12:1024-1032.

25. Miller SA, Dykes DD, Polesky HF. A Simple salting out procedure for extracting DNA from human nucleated cells (on-line). Nucleic Acids Res. 1988;16:1215. 
26. Zernant J, Külm M, Dharmaraj S, et al. Genotyping microarray (disease chip) for Leber congenital amaurosis: detection of modifier alleles. Invest Ophthalmol Vis Sci. 2005;46:3052-3059,

27. Perrault I, Hanein S, Gerber S, et al. Retinal dehydrogenase 12 (RDH12) mutations in Leber congenital amaurosis. Am J Hum Genet. 2004;75:639-646.

28. Freund CL, Wang QL, Chen SM, et al. De novo mutations in the CRX homeobox gene associated with Leber congenital amaurosis. Nat Genet. 1998;18:311-312.

29. Uvijls A, Leroy BP, Leys M, De Rouck A, Kestelyn P. A large field panel D-15 test for low vision patients. Bull Soc Belge Opbtalmol. 1998;270:57-65.

30. Dharmaraj S, Silva E, Pina AL, et al. Mutational analysis and clinical correlation in Leber congenital amaurosis. Opbthalmic Genet. 2000;21:135-150.

31. Felius J, Thompson DA, Khan NW, et al. Clinical course and visual function in a family with mutations in the RPE65 gene. Arch Ophthalmol. 2002;120:55-61.

32. Gu SM, Thompson DA, Srikumari CRS, et al. Mutations in RPE65 cause autosomal recessive childhood-onset severe retinal dystrophy. Nat Genet. 1997;17:194-197.
33. Hamel CP, Griffoin JM, Lasquellec L, Bazalgette C, Arnaud B. Retinal dystrophies caused by mutations in RPE65: assessment of visual functions. Br J Ophthalmol. 2001;85:424-427.

34. Lorenz B, Gyurus P, Preising M, et al. Early-onset severe rod-cone dystrophy in young children with RPE65 mutations. Invest Ophthalmol Vis Sci. 2000;41:2735-2742.

35. Yzer S, van den Born LI, Schuil J, et al. A Tyr368His RPE65 founder mutation is associated with variable expression and progression of early onset retinal dystrophy in 10 families of a genetically isolated population. J Med Genet. 2003;40:709713 .

36. Dharmaraj S, Leroy BP, Sohocki MM, et al. The phenotype of Leber congenital amaurosis in patients with AIPL1 mutations. Arch Ophthalmol. 2004;122:1029-1037.

37. Lotery AJ, Malik A, Shami SA, et al. CRB1 mutations may result in retinitis pigmentosa without para-arteriolar RPE preservation. $O p b$ thalmic Genet. 2001;22:163-169.

38. den Hollander AI, Davis J, van der Velde-Visser SD, et al. CRB1 mutation spectrum in inherited retinal dystrophies. Hum Mutat. 2004;24:355-369. 